\title{
LEM keeps the wheels turning
}

$\mathrm{CD}^{+} \mathrm{T}$ cells have a central role in defence against viruses and tumour cells, but chronic viral infections and high tumour load are associated with impaired $\mathrm{CD}^{+} \mathrm{T}$ cell population expansion and memory formation. Reporting in Science, Okoye et al. have identified an orphan protein which they have termed lymphocyte expansion molecule (LEM) - that promotes cytotoxic $\mathrm{T}$ lymphocyte

LEM promotes

CD8 + T cell

proliferation

... through the regulation of mitochondrial respiration

5

$\mathrm{T}$ cell formation.

The authors used a forwardgenetics approach with the chemical $N$-ethylnitrosourea (ENU) to generate a panel of 430 mutant mice. These mice were infected with a strain of lymphocytic choriomeningitis virus that causes a

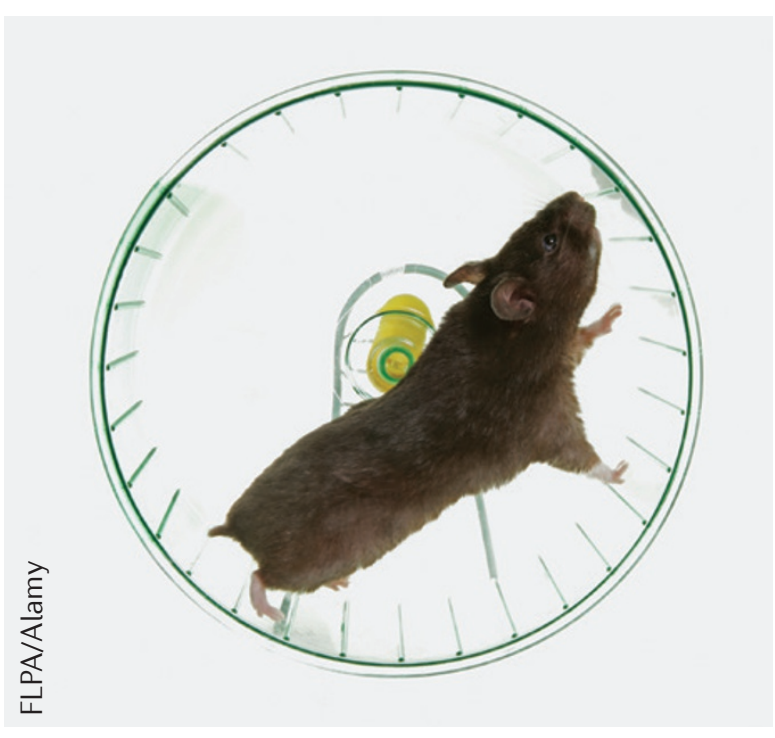

chronic infection, and the resulting antigen-specific CTL responses were assessed. From this analysis, the authors identified a mutant mouse strain of interest, which they termed the Retro mutant strain.

Chronically infected Retro mutant mice had a tenfold increase in virus-specific CTL numbers compared with infected wild-type mice. This resulted in lower viral loads in Retro mutant mice 8 days after infection through an increase in CTL-mediated immunity. Following acute viral infection, Retro mutant mice developed more central memory $\mathrm{CD}^{+} \mathrm{T}$ cells, as well as more short-lived effector cells, than wild-type mice. A similar increase in CTL numbers and killing of tumour cells was observed in Retro mutant mice injected with B16-F10 melanoma cells.

Using high-throughput exome sequencing, the authors found the Retro mutation to be an Ala to Gly transition of nucleotide 1304 in Bc055111 on chromosome 4, which encodes an orphan protein. They named this Bc055111-encoded protein LEM. The Ala1304Gly mutation stabilizes Lem mRNA in CTLs, resulting in increased protein expression. Together, these data suggest that LEM is a positive regulator of $\mathrm{CD}^{+} \mathrm{T}$ cell proliferation and memory formation that is upregulated in Retro mutant mice. Of note, C1ORF177 encodes the human homologue of LEM, and ectopic expression of human LEM in human $\mathrm{CD}^{+} \mathrm{T}$ cells increased their proliferation.

CR6-interacting factor 1 (CRIF1; also known as GADD45GIP1) is required for the translation and insertion of oxidative phosphorylation (OXPHOS) polypeptides into the inner membrane of the mitochondria. LEM was shown to interact with CRIF1, and this interaction within mitochondria controlled the activity of OXPHOS proteins. CTLs from infected Retro mutant mice had higher respiratory levels and increased levels of OXPHOS-dependent mitochondrial reactive oxygen species (mROS), whereas cells from LEMdeficient mice had decreased mROS, compared with cells from wild-type mice. Furthermore, inhibition of mROS in infected Retro mutant mice resulted in decreased CTL numbers and increased viral loads compared with wild-type mice.

Together, these data show that LEM promotes CD8 ${ }^{+} \mathrm{T}$ cell proliferation, effector function and memory formation through the regulation of mitochondrial respiration. Thus, therapeutically increasing the levels of active LEM may be useful in the treatment of chronic viral infections and cancer.

Olive Leavy

ORIGINAL RESEARCH PAPER Okoye, l. et al. The protein LEM promotes $C D 8^{+} T$ cell immunity through effects on mitochondrial respiration. Science http://dx.doi.org/10.1126/science.aaa7516 (2015) 